\title{
Electrical Stimulation Promotes Motoneuron Regeneration without Increasing Its Speed or Conditioning the Neuron
}

\author{
Thomas M. Brushart,, ${ }^{1,2}$ Paul N. Hoffman, ${ }^{2,3}$ Richard M. Royall, ${ }^{4}$ Beth B. Murinson, ${ }^{2}$ Christian Witzel, ${ }^{1}$ and \\ Tessa Gordon ${ }^{5}$ \\ Departments of ${ }^{1}$ Orthopaedics, ${ }^{2}$ Neurology, and ${ }^{3}$ Opthalmology, Johns Hopkins School of Medicine, and Department of \\ ${ }^{4}$ Biostatistics and Statistics, Bloomberg School of Public Health, Johns Hopkins University, Baltimore Maryland 21287, \\ and ${ }^{5}$ Department of Pharmacology, Division of Neuroscience, University of Alberta, Alberta T6G 2S2, Canada
}

\begin{abstract}
Motoneurons reinnervate the distal stump at variable rates after peripheral nerve transection and suture. In the rat femoral nerve model, reinnervation is already substantial 3 weeks after repair, but is not completed for an additional 7 weeks. However, this "staggered regeneration" can be temporally compressed by application of $20 \mathrm{~Hz}$ electrical stimulation to the nerve for $1 \mathrm{hr}$. The present experiments explore two possible mechanisms for this stimulation effect: (1) synchronization of distal stump reinnervation and (2) enhancement of regeneration speed. The first possibility was investigated by labeling all motoneurons that have crossed the repair at intervals from $4 \mathrm{~d}$ to 4 weeks after rat femoral nerve transection and suture. Although many axons did not cross until 3-4 weeks after routine repair, stimulation significantly increased the number crossing at 4 and $7 \mathrm{~d}$, with only
\end{abstract}

a few crossing after 2 weeks. Regeneration speed was studied by radioisotope labeling of transported proteins and by anterograde labeling of regenerating axons, and was not altered by stimulation. Attempts to condition the neuron by stimulating the femoral nerve 1 week before injury were also without effect. Electrical stimulation thus promotes the onset of motor axon regeneration without increasing its speed. This finding suggests a combined approach to improving the outcome of nerve repair, beginning with stimulation to recruit all motoneurons across the repair, followed by other treatments to speed and prolong axonal elongation.

Key words: electrical stimulation; peripheral nerve; anterograde tracing; BDNF; conditioning; neurobiotin
In the mid-eighteenth century, electrical stimulation became a popular treatment for disorders of the nervous system (Du BoisReymond, 1848; Duchenne de Boulogne, 1855). Although an aura of charlatinism hung over this activity for many years, recent work has demonstrated its potential. Peripheral nerve regeneration has been manipulated with weak DC currents, pulsed electromagnetic fields (PEMFs), and alternating currents strong enough to discharge the neuron. Weak DC currents orient and direct regeneration in vitro (Hinkle et al., 1981; Patel and Poo, 1982) but produce inconsistent results in vivo (Politis et al., 1988; Kerns et al., 1991, 1994; Kerns and Lucchinetti, 1992). PEMF, attractive because of its noninvasive nature, increases the speed of axon regeneration in the first week by $22-24 \%$ (Sisken et al., 1989; Rusovan et al., 1992) but without improving functional outcome (Orgel et al., 1984; Zienowicz et al., 1991). Against this background, use of brief current pulses to repeatedly discharge the parent neurons has emerged as a promising option (Nix and Hopf, 1983; Pockett and Gavin, 1985).

We have studied the effects of brief electrical stimulation in the rat femoral nerve model (Brushart, 1988). Proximally, at the site of nerve transection and repair, axons destined for skin and muscle intermingle within the nerve trunk. Regenerating motor

\footnotetext{
Received Nov. 5, 2001; revised April 29, 2002; accepted May 20, 2002.

This work was supported by National Institutes of Health Grant NS34484 (T.M.B.) and the Spinal Cord Research Fund of the PVA (P.H.). We thank Philip Kessens for technical assistance, Kate Weaver for artwork, Norman Barker for photography, and Dr. Pamela Talalay for editorial help.

Correspondence should be addressed to Dr. Thomas M. Brushart, Johns Hopkins Orthopaedics, 601 North Caroline Street, Baltimore, MD 21287. E-mail: tbrusha@jhmi.edu.

Copyright (C) 2002 Society for Neuroscience $\quad 0270-6474 / 02 / 226631-08 \$ 15.00 / 0$
}

axons that contact the distal stump will thus have access to Schwann cell tubes that lead either to muscle or to skin. Distally, the nerve bifurcates into a muscle branch to the quadriceps and a purely cutaneous branch. The specificity of regeneration is assessed by separately labeling and counting motoneurons that have projected axons correctly to the muscle branch or incorrectly to the cutaneous branch.

When the nerve is transected and sutured, reinnervation of the distal stump occurs gradually (Brushart, 1990, 1993; Al-Majed et al., 2000b). At 2 weeks, equal numbers of motoneurons project correctly to muscle and incorrectly to skin. Between 2 and 10 weeks, the number of incorrect projections remains constant while the number of correct projections gradually increases, demonstrating a process of staggered regeneration. This "stagger" is dramatically compressed, however, by application of $20 \mathrm{~Hz}$ electrical stimulation to the site of nerve repair for $1 \mathrm{hr}$ (Al-Majed et al., 2000b). As a result, reinnervation 3 weeks after stimulation and repair is equal to that requiring 8-10 weeks after suture without stimulation.

The current experiments examine two potential mechanisms for the reduction of regeneration "stagger" by electrical stimulation: synchronization of growth across the nerve repair, or increase in the speed of motor axon regeneration within the distal stump. To examine the former possibility, our standard labeling procedure was altered to permit identification of motor axons as soon as they have crossed the repair and entered the distal stump. Regeneration speed was studied by radioisotope labeling of transported proteins and by anterograde labeling of regenerating axons. Intraoperative stimulation promoted distal stump reinnervation, but had no effect on regeneration speed. Similarly, no 


\begin{tabular}{|c|c|c|c|}
\hline Experimental group & Manipulation & Technique of evaluation & Nerves per subgroup \\
\hline \multirow[t]{2}{*}{ Repair crossing } & Repair & Proximal labeling & $\begin{array}{l}4 \mathrm{~d}(n=12), 1 \text { week }(n=10), 2 \text { weeks }(n=9), \\
3 \text { weeks }(n=10), 4 \text { weeks }(n=10)\end{array}$ \\
\hline & Repair + stimulation & Proximal labeling & $\begin{array}{l}4 \mathrm{~d}(n=12), 1 \text { week }(n=10), 2 \text { weeks }(n=9), \\
3 \text { weeks }(n=10), 4 \text { weeks }(n=10)\end{array}$ \\
\hline \multirow[t]{2}{*}{ Proximal labeling control } & Repair & Proximal labeling & Immediate $(n=6)$ \\
\hline & Normal nerve & Proximal labeling & Immediate $(n=6)$ \\
\hline \multirow[t]{7}{*}{ Regeneration speed } & Crush & Radiolabel & Label $3 \mathrm{~d}$ after crush, count $4 \mathrm{~d}$ after $\operatorname{crush}(n=5)$ \\
\hline & Crush + stimulation & Radiolabel & Label $3 \mathrm{~d}$ after crush, count $4 \mathrm{~d}$ after crush $(n=5)$ \\
\hline & Crush & Radiolabel & Label $7 \mathrm{~d}$ after crush, count $8 \mathrm{~d}$ after crush $(n=5)$ \\
\hline & Crush + stimulation & Radiolabel & Label $7 \mathrm{~d}$ after crush, count $8 \mathrm{~d}$ after crush $(n=5)$ \\
\hline & Repair & Anterograde labeling & Label $4 \mathrm{~d}$ after repair $(n=5)$ \\
\hline & Repair + stimulation & Anterograde labeling & Label 4 d after repair $(n=5)$ \\
\hline & Normal nerve & Anterograde labeling & Immediate $(n=4)$ \\
\hline \multirow[t]{3}{*}{ Conditioning trial } & Repair & Distal labeling & 3 weeks $(n=11)$ \\
\hline & Stimulation, repair $7 \mathrm{~d}$ later & Distal labeling & 3 weeks $(n=11)$ \\
\hline & Stimulation, crush $7 \mathrm{~d}$ later & Radiolabel & Label $7 \mathrm{~d}$ after crush, count $8 \mathrm{~d}$ after $\operatorname{crush}(n=6)$ \\
\hline
\end{tabular}

conditioning effect was observed if stimulation was performed 1 week before the crush was delivered. Electrical stimulation thus recruits motoneurons to cross the repair site and enter the distal stump through a mechanism separate from that resulting in early or "conditioned" increases in regeneration speed. This finding suggests a combined approach to improving the outcome of nerve repair, beginning with stimulation to recruit all motoneurons across the repair, followed by other treatments to speed and prolong axonal elongation.

\section{MATERIALS AND METHODS}

Experiments were performed on the femoral nerves of young adult (250 gm) female Sprague Dawley rats. The animals were anesthetized by intramuscular injection of ketamine $(87 \mathrm{mg} / \mathrm{kg})$ and xylazine $(13 \mathrm{mg} / \mathrm{kg})$. Four groups of animals were prepared (Table 1). The repair crossing group evaluated the effect of electrical stimulation on the timing of distal stump penetration by regenerating motor axons. Both femoral nerves of each animal were transected and repaired, but only one repair was stimulated. Repairs were evaluated by proximal labeling (Fig. 1) at $4 \mathrm{~d}$ $(n=12$ rats), 1 week $(n=10), 2$ weeks $(n=9), 3$ weeks $(n=10)$, and 4 weeks $(n=10)$ after surgery. Proximal labeling controls $(n=6$ rats $)$ were evaluated to rule out spurious labeling of proximal stump axons by diff usion of tracer and to establish the number of motoneurons normally available for regeneration at this level of the femoral nerve. One femoral nerve was transected and repaired followed by immediate proximal labeling; the opposite nerve was labeled at the same level. The regeneration speed groups were designed to assess the speed of motor axon regeneration within the distal stump. In radiolabeled animals $\left[{ }^{35} \mathrm{~S}\right] \mathrm{me}-$ thionine was injected into motoneurons 3 or $7 \mathrm{~d}$ after crush to localize the peak and front of advancing axons $4 \mathrm{~d}$ (crush, $n=5$; crush + stimulation, $n=5$ ) or $8 \mathrm{~d}$ (crush, $n=5$; crush + stimulation, $n=5$ ) after injury. Regeneration speed was also examined by anterograde tracing of regenerating axons $4 \mathrm{~d}$ after femoral nerve repair $(n=5)$ or repair with stimulation $(n=5)$. Four normal nerves were studied to determine the maximum distance over which axons could be traced with this technique. The conditioning trial groups examined the possibility that electrical stimulation might condition the neuron to respond to subsequent injury with more rapid axon elongation. Eleven rats underwent bilateral femoral nerve exposure and unilateral stimulation (the potential conditioning lesion), followed in 1 week by bilateral transection and repair. After 3 weeks of regeneration, motoneurons reinnervating the femoral cutaneous and muscle branches were evaluated with routine distal labeling (Fig. 1). In additional animals $(n=6)$, unilateral femoral exposure and stimulation were followed in 1 week by nerve crush and evaluated by $\left.{ }^{35} \mathrm{~S}\right]$ methionine injection $7 \mathrm{~d}$ after crush and counting $1 \mathrm{~d}$ later.

Nerve repair and stimulation. The femoral nerve was sharply transected $1 \mathrm{~mm}$ distal to the iliacus branch, carefully aligned, and sutured with
11-0 nylon under $20-40 \times$ magnification. When required, stimulation was delivered intraoperatively for $1 \mathrm{hr}$ with animals under the same anesthetic. A Grass (Quincy, MA) SD-9 stimulator delivered continuous $20 \mathrm{~Hz}$ stimulation $(100 \mu \mathrm{sec}, 3-5 \mathrm{~V})$ to fine silver wires placed at (anode) and just proximal to (cathode) the repair.

Proximal and distal labeling of motoneurons. Proximal labeling was used to identify motoneurons as soon as possible after their axons entered the distal nerve stump (Fig. 1). The femoral nerve was exposed and crushed $1.5 \mathrm{~mm}$ distal to the suture line with narrow microforceps. A micropipette was then introduced through the epineurium and perineurium distal to the crush and advanced intraneurally to the crush zone. We injected $\sim 0.5 \mu \mathrm{l}$ of $5 \%$ Fluororuby (FR; Molecular Probes, Eugene, OR) with a Picospritzer (Parker Hannefin, Fairfield, NJ) to restore the flattened crush zone to its normal rounded contour. Forty-eight hours later the animals were deeply anesthetized before being perfused through the left ventricle. A warm saline flush $(150 \mathrm{ml})$ was followed by $500 \mathrm{ml}$ of $4 \%$ paraformaldehyde in $0.1 \mathrm{M}$ Sorensen's phosphate buffer. Postfixation in the same solution overnight followed by immersion in $20 \%$ sucrose, also in $0.1 \mathrm{M}$ Sorensen's phosphate buffer, prepared the tissue for sectioning. Forty micrometer sections were cut on a freezing microtome, serially mounted on glass slides, dried, and overlaid with coverslips using DPX (Aldrich, Milwaukee, WI) to minimize extraneous fluorescence.

Distal labeling was performed to assess the potential for electrical stimulation to act as a "conditioning" lesion (Fig. 1). This technique separately identifies motoneurons projecting correctly to the quadriceps muscle, incorrectly to the saphenous nerve, or simultaneously to both (double-labeled). The muscle branch is severed as it enters the quadriceps muscle; the cutaneous branch is cut an equivalent distance from the femoral bifurcation to produce proximal cutaneous and muscle stumps of equal length. One stump (randomly chosen) is exposed to 3\% Fluoro Gold (FG; Fluorochrome, Denver, CO) for $1 \mathrm{hr}$ in a well of petroleum jelly, after which it is copiously irrigated and loosely sutured to a distant portion of the wound. The other stump is then exposed to 5\% FR for 1 hr, similarly irrigated, and sewn to the opposite corner of the wound to prevent cross-contamination by diffusion of tracers. Subsequent preparation follows the protocol for proximal labeling given above.

Motoneuron counting and data analysis. Spinal cord sections were viewed with fluorescent light (FG, $323 \mathrm{~nm}$ excitation, $408 \mathrm{~nm}$ emission; FR, $555 \mathrm{~nm}$ excitation, $580 \mathrm{~nm}$ emission) by an observer unaware of the experimental treatment. All labeled motoneurons were counted, and the presence of split cells was corrected for as described by Abercrombie (1946). The validity of this approach has been discussed previously (Brushart et al., 1998). Results in the repair crossing group were first used to determine the mean total number of motoneurons that had crossed the repair at each time period in stimulated and nonstimulated groups. These means were subjected to $t$ test comparison. The reinnervation process was then further characterized by using this data to calculate the number of new motoneurons crossing the repair at each interval between labeling times. This value was obtained by subtracting 

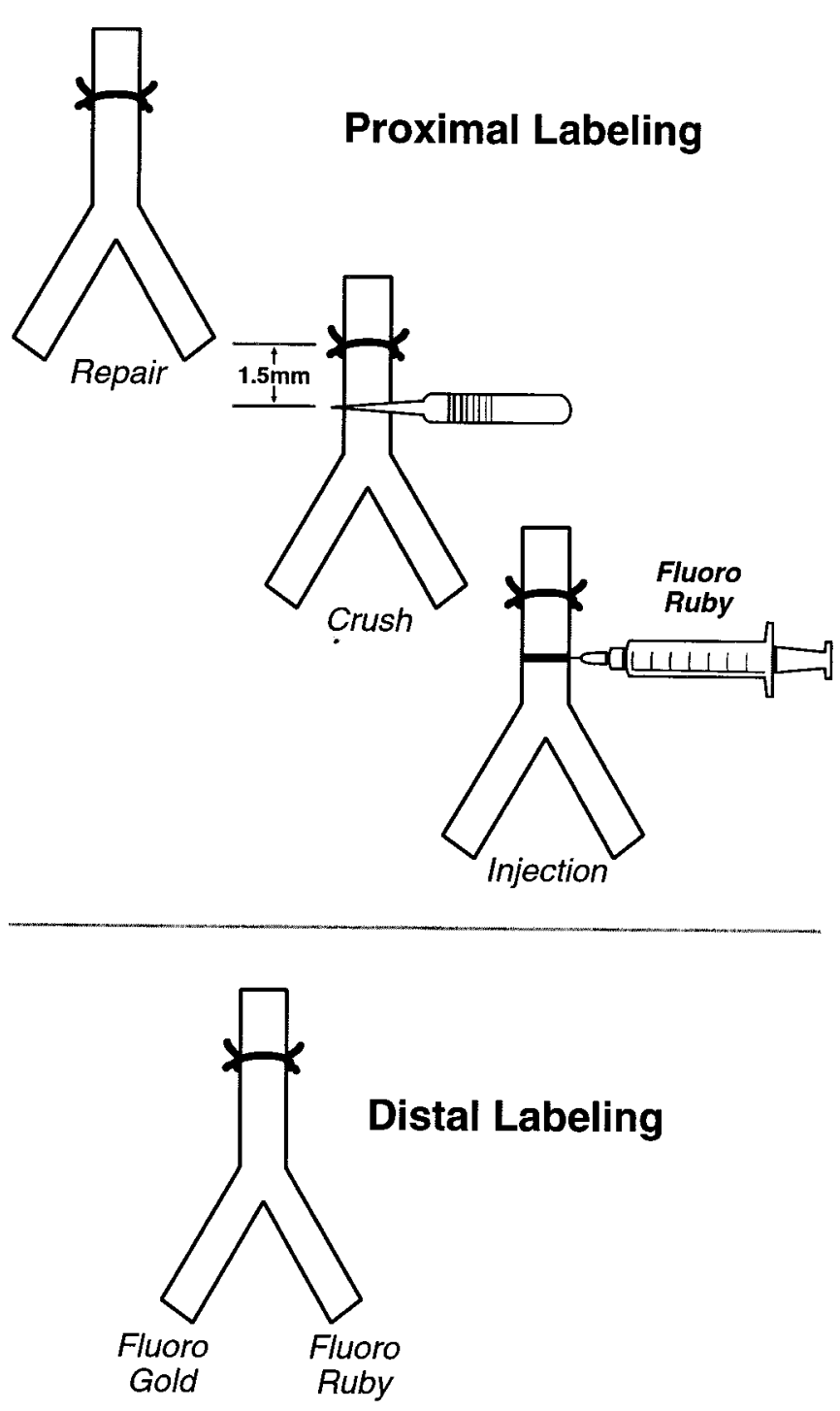

Figure 1. Proximal and distal labeling. Proximal labeling identifies motor axons as soon as possible after they have entered the distal stump, but it does not differentiate between cutaneous and muscle pathways. Distal labeling is performed after axons have been segregated into cutaneous and muscle branches, revealing their destination but not their early behavior.

the mean number of motoneurons labeled at the beginning of the interval from the mean number labeled at the end of the interval for both stimulated and nonstimulated groups. The resulting means were also subjected to $t$ test comparison.

In the conditioning trial each femoral motoneuron pool was counted for: (1) FR-labeled motoneurons, (2) FG-labeled motoneurons, and (3) double-labeled motoneurons. Each group was then characterized by three means: the mean number of motoneurons projecting correctly to the muscle branch, the mean number projecting incorrectly to the cutaneous branch, and the mean number of "double-labeled" neurons, those projecting axon collaterals to both branches. A standard two-sample $t$ test was used to compare counts of motoneurons projecting to cutaneous and muscle branches within each group and to muscle or cutaneous branches between the groups.

Radiolabeling experiments. In the regeneration speed experiments, fast axonal transport was used to determine the locations of regenerating motor fibers either 4 or $8 \mathrm{~d}$ after regeneration was initiated by crushing the sciatic nerve, with or without electrical stimulation (Forman and Berenberg, 1978). Crush was chosen to initiate regeneration to eliminate the confounding effects of regeneration stagger that accompany nerve transection and suture. Conditioning trial animals received stimulation 1 week before crush and analysis $8 \mathrm{~d}$ after crush. All experiments were performed in 7-week-old Sprague Dawley rats to standardize the rate of axoplasmic transport. The sciatic nerve was crushed $5 \mathrm{~mm}$ distal to the hamstring branch twice for $30 \mathrm{sec}$ with microforceps, and the site was marked with a 10-0 nylon suture. Either 3 or $7 \mathrm{~d}$ after crush, motor neurons were labeled at four sites along the L4 and L5 levels of the spinal cord by injecting $1 \mu \mathrm{l}$ of $\left[{ }^{35} \mathrm{~S}\right]$ methionine $(50 \mu \mathrm{Ci} / \mu \mathrm{l})$ at each site (Lasek, 1968). Animals were killed $1 \mathrm{~d}$ after labeling, (4 or $8 \mathrm{~d}$ after crush). Sciatic nerves were removed and cut into consecutive 3-mm-long segments so that the $1-\mathrm{mm}$-wide crush site was located $2 \mathrm{~mm}$ from the proximal end of its segment. Each segment was dissolved in $100 \mu \mathrm{l}$ of 2 $\mathrm{N} \mathrm{NaOH}$ at $60^{\circ} \mathrm{C}$ for $1 \mathrm{hr}$. Samples were cooled and neutralized with 100 $\mu \mathrm{l}$ of $2 \mathrm{~N} \mathrm{HCl}$ before levels of radioactivity were measured by liquid scintillation spectroscopy. Mean values and SEs were plotted as a function of distance from the crush site. The distance from the crush at which the level of radioactivity in the leading edge of the curve fell to $50 \%$ of mean peak value was used to characterize each nerve. Means were determined for stimulated and nonstimulated nerves at 4 and $8 \mathrm{~d}$ and were subjected to $t$ test comparison. The mean rate of regeneration was calculated for stimulated and nonstimulated groups with the equation: rate $=$ (mean location of the leading edge at $8 \mathrm{~d}$ minus mean location of the leading edge at $4 \mathrm{~d}) / 4 \mathrm{~d}$.

Anterograde tracing. The distal extent of axon regeneration was measured by anterograde tracing $4 \mathrm{~d}$ after nerve suture to correspond to the earliest group in the proximal labeling studies. Axons were labeled by crushing the nerve $2 \mathrm{~mm}$ proximal to the repair and injecting the crush site with 2.5\% Neurobiotin (Vector Laboratories, Burlingame, CA). Four hours were allowed for anterograde transport, after which the animals were perfused for 30 min with $4 \%$ paraformaldehyde in $0.1 \mathrm{M}$ Sorensen's phosphate buffer. The nerves were removed, post-fixed in the perfusate, and stored in $20 \%$ sucrose in Sorensen's phosphate buffer. They were then embedded in $10 \%$ gelatin, $10 \%$ sucrose, and sectioned longitudinally at $60 \mu \mathrm{m}$ on a sliding microtome.

Tissue sections were rinsed in PBS ( $3 \times 10 \mathrm{~min})$, blocked with $50 \%$ methanol containing $0.4 \%$ Triton X-100 and 3.3\% hydrogen peroxide (1 $\mathrm{hr}$ ), and rinsed again before soaking in serum-free blocking agent (Dako, Carpenteria, CA; $1 \mathrm{hr}$ ). A third rinse was followed by incubation in streptavidin labeled with Cy3 [Jackson ImmunoResearch, West Grove, PA; absorption (abs) 550, emission (em) 570] diluted 1:500 in PBS for 1 $\mathrm{hr}$ and a final rinse in PBS. Some specimens were also processed to demonstrate laminin with a second fluorescent label. These were transferred directly to serum-free blocking agent, then reacted overnight with antibodies to laminin (Sigma, St. Louis, MO; L-9393) diluted 1:250 in serum-free blocking agent. The following morning sections were treated with Alexa Fluor 488 (Molecular Probes; abs 495, em 518) diluted 1:300 for $2 \mathrm{hr}$ before a final rinse with PBS. Sections were mounted on glass slides, coverslipped with DPX, and viewed with a Nikon Optiphot fluorescent microscope. A calibrated reticule was used to measure the farthest distance traveled by at least five axons in each nerve.

\section{RESULTS}

\section{Repair crossing}

Proximal labeling was used to evaluate 102 nerve repairs in the 51 animals of the repair crossing group. All animals underwent bilateral repair with unilateral stimulation. Evaluation was performed at $4 \mathrm{~d}(n=12$ animals $), 1$ week $(n=10), 2$ weeks $(n=9)$, 3 weeks $(n=10)$, and 1 month $(n=10)$. The results of this evaluation are first presented as the total number of motoneurons labeled at each time period in stimulation (stim) and control (cont) groups (Fig. 2). Even at $4 \mathrm{~d}$, more motoneurons have crossed the repair site to reinnervate the distal stump in the stimulated group (stim, 40; cont, $13 ; p=0.02$ ). Significantly more motoneurons are labeled after stimulation at subsequent time periods $(p<0.003)$ until 4 weeks, when the curves converge $(p=$ 0.14). Stimulation thus increases distal stump reinnervation throughout the 3 weeks after nerve transection and repair.

Greater insight into the effects of electrical stimulation on distal stump reinnervation results from identification of the new "crossing events" that occur during each interval between labeling times (Fig. 3). This value was obtained by subtracting the 


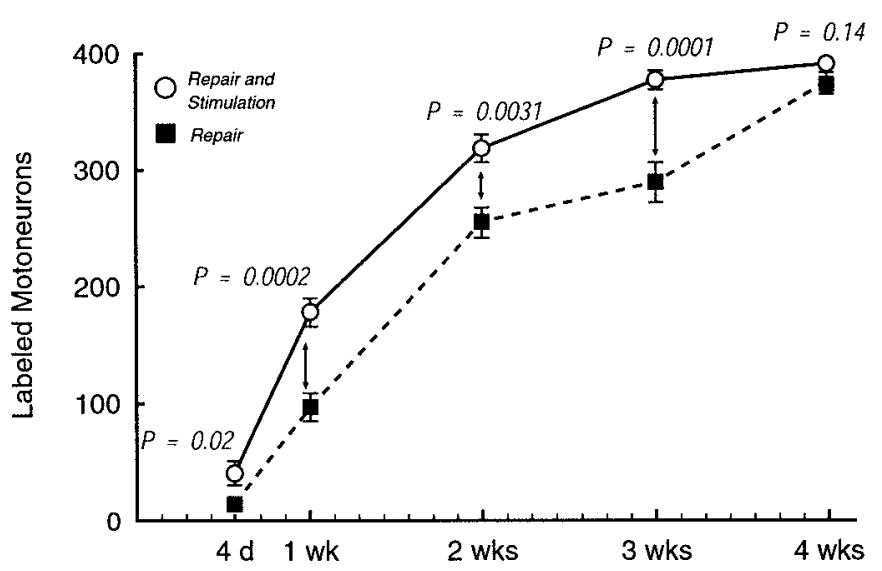

Figure 2. Cumulative distal stump reinnervation. The mean total numbers of motoneurons that have crossed the repair site with and without stimulation are plotted as a function of time after repair. Significantly more motoneurons have crossed in the stimulated nerves at all times during the first 3 weeks.

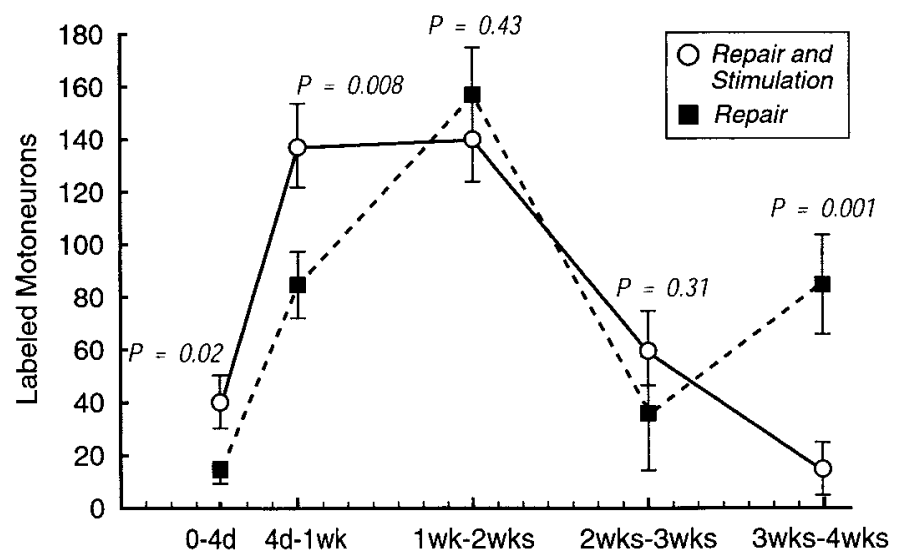

Figure 3. New crossing events. Each value was obtained by subtracting the mean number of motoneurons labeled at the beginning of an interval from the mean number labeled at the end of the interval for both stimulated and nonstimulated groups. The means at the $0-4 \mathrm{~d}$ interval are essentially the $4 \mathrm{~d}$ means, because a mean of only two motoneurons were labeled at the time of repair. The means at $4 \mathrm{~d}$ to 1 week in this figure represent the total number of motoneurons labeled at 1 week (Fig. 2) less the $4 \mathrm{~d}$ values, and so forth for each interval. Stimulation causes a rapid increase in crossing events to a peak between 1 and 2 weeks, with a more gradual decline. In contrast, the control curve is biphasic with a more gradual rise to an initial peak at 2 weeks, followed by rapid decline and then a second peak at 4 weeks. Electrical stimulation thus recruits many motoneurons to regenerate across the suture line and penetrate the distal stump earlier than they normally would.

mean number of motoneurons labeled at the beginning of the interval from the mean number labeled at the end of the interval for both stimulated and nonstimulated groups. In the first $4 \mathrm{~d}$ of the experiment, stimulation increases the mean number of motoneurons crossing the repair from 13 to $40(p=0.02)$. Between $4 \mathrm{~d}$ and 1 week, 137 new motoneurons enter the distal stump after stimulation, whereas only 84 enter in nonstimulated controls $(p=$ $0.008)$. Between 1 and 2 weeks, the number of crossings after stimulation remains constant $(n=140)$, whereas the controls rise to a similar level $(n=157 ; p=0.43)$. New crossing events are much less frequent in the 2-3 week interval in both groups (stim 60 , cont $35 ; p=0.31$ ). However, a significant difference again emerges between 3 and 4 weeks as the stimulated group decreases

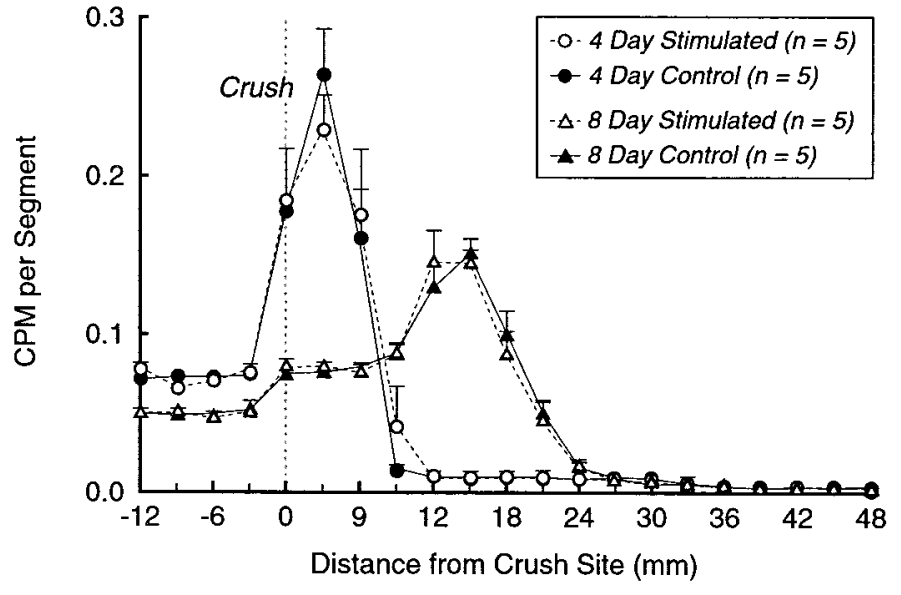

Figure 4. Regeneration speed. Sciatic nerve crush with or without stimulation was followed in 3 or $7 \mathrm{~d}$ by injection of $\left[{ }^{35} \mathrm{~S}\right]$ methionine into the parent motoneuron pool, and counts were obtained from peripheral nerve at 4 and $8 \mathrm{~d}$, respectively. Counts per minute are plotted as a function of distance from the crush site. Each curve represents the mean of five nerves, normalized to facilitate comparison. Electrical stimulation had no effect on regeneration speed at either 4 or $8 \mathrm{~d}$ after crush.

further toward the baseline, whereas a fresh wave of crossings is seen in the controls (stim 14 , cont $84 ; p=0.001$ ). In comparing the two curves (Fig. 3), stimulation causes a rapid increase to a peak between 1 and 2 weeks, with a more gradual decline. In contrast, the control curve is biphasic with a more gradual rise to an initial peak at 2 weeks, followed by rapid decline and then a second peak at 4 weeks. Electrical stimulation thus profoundly alters the response to nerve transection and repair by recruiting many motoneurons to regenerate across the suture line and penetrate the distal stump earlier than they normally would.

\section{Proximal labeling controls}

The proximal labeling controls confirmed the effectiveness of this technique. Injection of FR immediately after repair in six nerves labeled a mean of only two motoneurons, eliminating the possibility that diffusion of tracer across the suture line labeled proximal stump axons in significant numbers. Injection of uninjured nerve labeled a mean of 412 motoneurons, an increase over the number labeled distally from the quadriceps branch in previous experiments because of the intervening branch to pectineus (Brushart et al., 1998).

\section{Regeneration speed-radiolabeling}

Data from the regeneration speed experiments are summarized in Figure 4. The distribution of counts obtained 4 and $8 \mathrm{~d}$ after injury are strikingly similar for stimulated and nonstimulated groups. These data were further analyzed by characterizing each experimental nerve by the distance from the crush at which the level of radioactivity in the leading edge of the curve fell to $50 \%$ of the mean peak value. Means of this value were obtained for each group: $4 \mathrm{~d}$ control, $7.05 \pm 0.93 \mathrm{~mm} ; 4 \mathrm{~d}$ stim, $6.85 \pm 0.55$ $\mathrm{mm} ; 8 \mathrm{~d}$ control, $20.14 \pm 0.86 \mathrm{~mm} ; 8 \mathrm{~d}$ stim, $20.36 \pm 0.62 \mathrm{~mm}$. Comparison of these means revealed no significant differences at either $4(p=0.858)$ or $8(p=0.837) \mathrm{d}$, providing no evidence for a significant stimulation effect on regeneration speed. Calculation of mean regeneration speed from these values yielded a speed of $3.38 \pm 0.58 \mathrm{~mm} / \mathrm{d}$ with stimulation and $3.27 \pm 0.90 \mathrm{~mm} / \mathrm{d}$ without stimulation. 

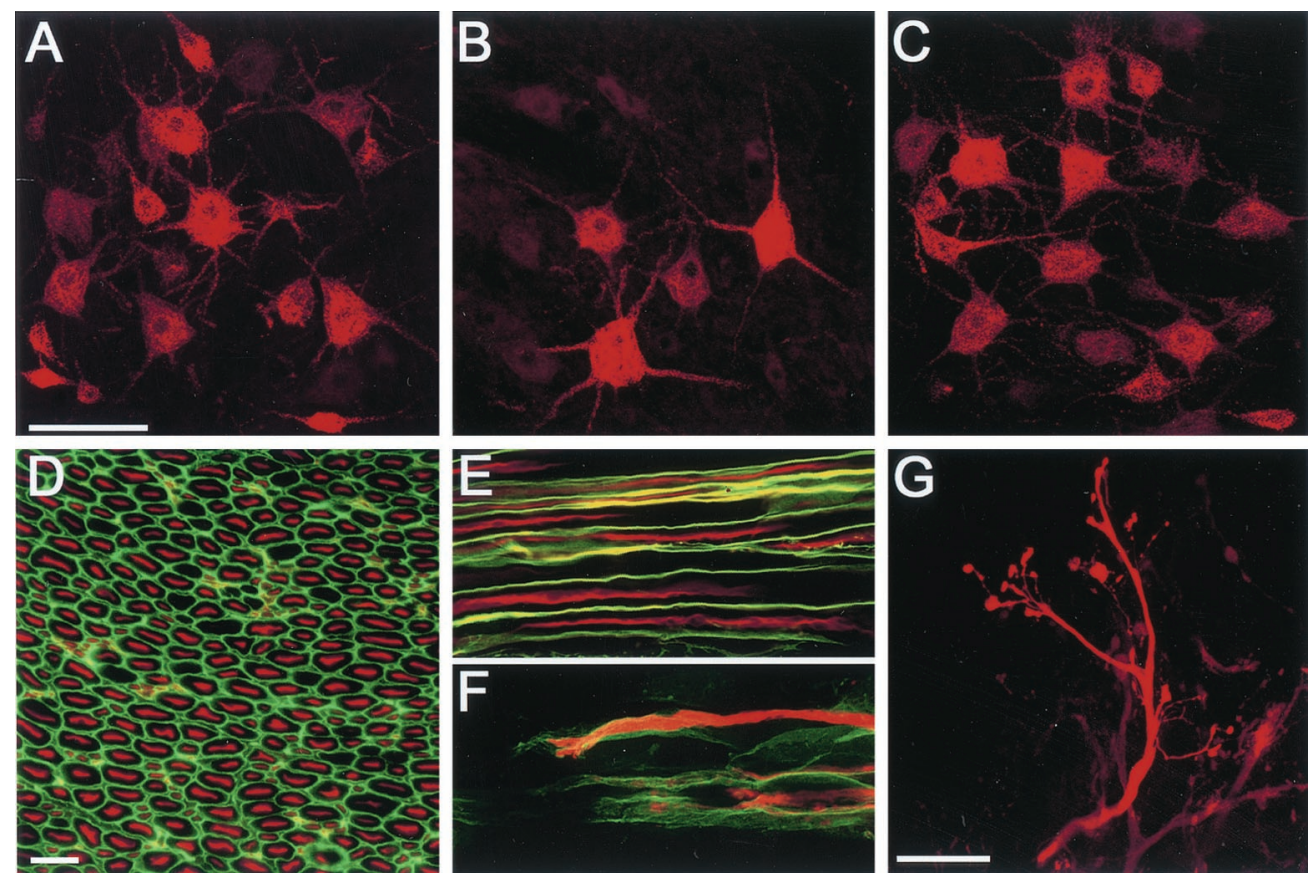

Figure 5. Confocal microscopy of labeled motoneurons and axons. $A-C$ represent femoral motoneurons retrogradely labeled with Fluoro Ruby (Scale bar, $100 \mu \mathrm{m})$. $A$, Normal femoral motoneuron pool; $B$, femoral motoneuron pool 1 week after nerve repair without stimulation. Motoneurons were labeled by crushing the nerve $1.5 \mathrm{~mm}$ distal to the repair and injecting the crush site with Fluoro Ruby (proximal labeling technique, Fig. 1). This technique labels axons as soon as they have entered the distal stump. $C$, Femoral motoneuron pool 1 week after nerve repair with stimulation, demonstrating an increase in the number of motoneurons that have crossed the repair site. $D-F$, Peripheral nerve double-labeled by anterograde transport of Neurobiotin (red) and antibodies to laminin (green) (Scale bar, $10 \mu \mathrm{m}$ ). D, Normal nerve $8 \mathrm{~mm}$ distal to the injection site, demonstrating nearly complete labeling of the nerve. $E$, Longitudinal section of normal nerve. The myelin sheath remains unlabeled. $F$, Axon reinnervating the distal stump. The axon is closely opposed to the basement membrane and is clearly identifiable by its growth cone. The Schwann cell tube below the axon contains fragmented diffusion artifact, which is confusing when viewed in cross-section but easily identified by its contour on longitudinal section. $G$, Prof usely branched axon with multiple growth cones crossing the repair site of a stimulated animal. The axon is labeled with Neurobiotin only (Scale bar, $50 \mu \mathrm{m})$.

\section{Regeneration speed-anterograde tracing}

Four days after nerve repair without stimulation, regenerating axons had traveled a mean of $3.38 \mathrm{~mm}$ (range, $2.8-4.0 \mathrm{~mm}$ ) (Fig. 5). After stimulation, they traveled a mean of $3.16 \mathrm{~mm}$ (range, 2.8-3.8 mm). Stimulation thus failed to increase regeneration speed ( $p=0.4298)$. Labeled axons could be traced for $10-12 \mathrm{~mm}$. from the site of Neurobiotin injection in normal nerve, confirming the effectiveness of our technique for the study of early regeneration.

\section{Conditioning trial}

The conditioning trial experiments investigated the possibility that stimulation might condition the neuron to elongate more rapidly after subsequent injury. A potential conditioning effect was sought by distal labeling after femoral nerve suture (Fig. 6) and with transport studies after sciatic crush (Fig. 7). Stimulation 1 week before femoral nerve suture had no significant effect on either the number of motoneurons that correctly reinnervated the muscle branch (stim, 112; sham stim, $115 ; p=0.86$ ) or that incorrectly reinnervated the cutaneous branch (stim, 105; sham stim, 124; $p=0.40$ ). Previous stimulation thus had no effect on the outcome of reinnervation in this model. Similarly, the speed of regeneration $8 \mathrm{~d}$ after sciatic crush was not altered by stimulation 1 week before the injury, because the location of the 50\% mean peak value did not differ significantly from that in controls (conditioning trial, $20.86 \pm 0.31$; control, $20.14 \pm 0.86 ; p=0.39$ ). In aggregate, these experiments establish that stimulation alone has no significant impact on the outcome of regeneration when the nerve is subsequently injured, and thus does not serve as a "conditioning" lesion.

\section{DISCUSSION}

\section{Staggered regeneration}

Attempts to quantify axon behavior after peripheral nerve transection and repair have focused on the number of axons regenerating (Jenq and Coggeshall, 1984; Scherer and Easter, 1984; Mackinnon et al., 1991), their speed (Forman and Berenberg, 1978; Forman et al., 1979; Danielsen et al., 1986; Jacquin et al., 1992), and their initial delay at the suture line (Forman et al., 1979; Danielsen et al., 1986; Holmquist et al., 1993). The asynchrony of axon regeneration, which we have termed "staggered regeneration," has received little attention. Axon counts alone cannot detect regeneration stagger because of the multiple sprouts generated by each regenerating axon (for review, see Brushart, 1993). An identical picture would result if all proximal axons penetrated the distal stump with two collaterals, or half the proximal axons failed to regenerate and the other half generated four collaterals each. Similarly, studies of regeneration speed or suture line delay that identify only the fastest growing axons (Forman et al., 1979; Jacquin et al., 1992; Holmquist et al., 1993) will ignore those left behind. However, use of radiotracers to pinpoint the location of growth cones throughout the nerve should be more informative. In fact, both studies of transected and sutured nerve (Forman and Berenberg, 1978; Danielsen et al., 1986) identified substantial numbers of axons that failed to 


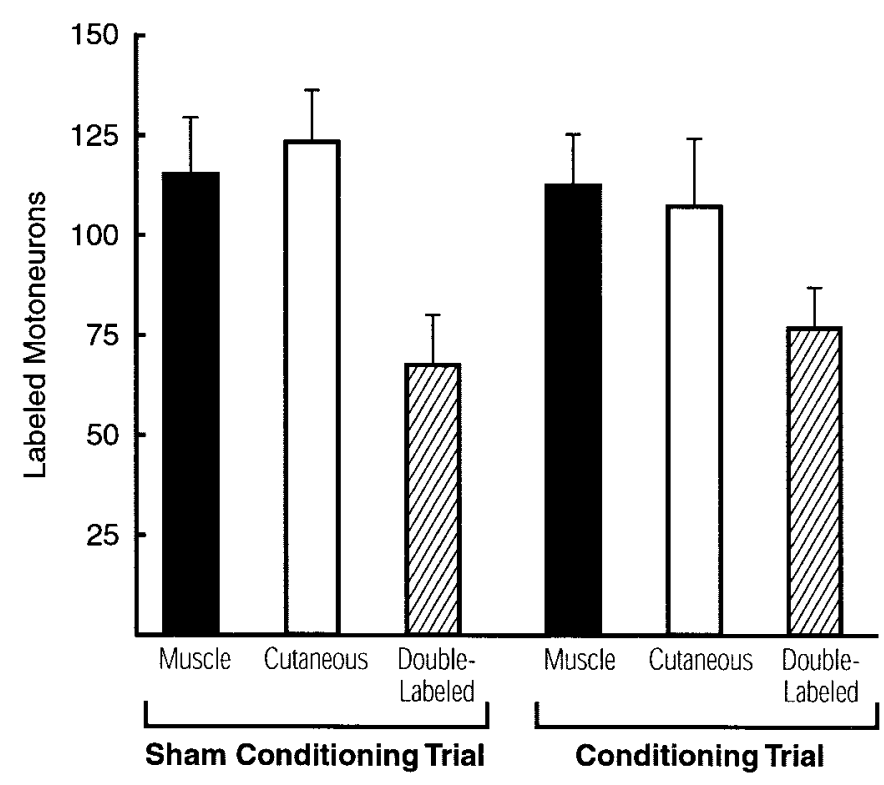

Figure 6. Conditioning trials-distal labeling. The femoral nerve was exposed bilaterally and stimulated unilaterally in 11 rats. One week later, both femoral nerves were transected and repaired. After allowing 3 weeks for regeneration, results were evaluated with distal labeling. For both groups of 11 nerves, the black bar represents the mean number of motoneurons projecting correctly to the muscle branch, the white bar represents the mean number projecting incorrectly to the cutaneous branch, and the striped bar represents the mean number projecting to both branches (double-labeled). Stimulation 1 week before repair had no effect on the outcome of regeneration.

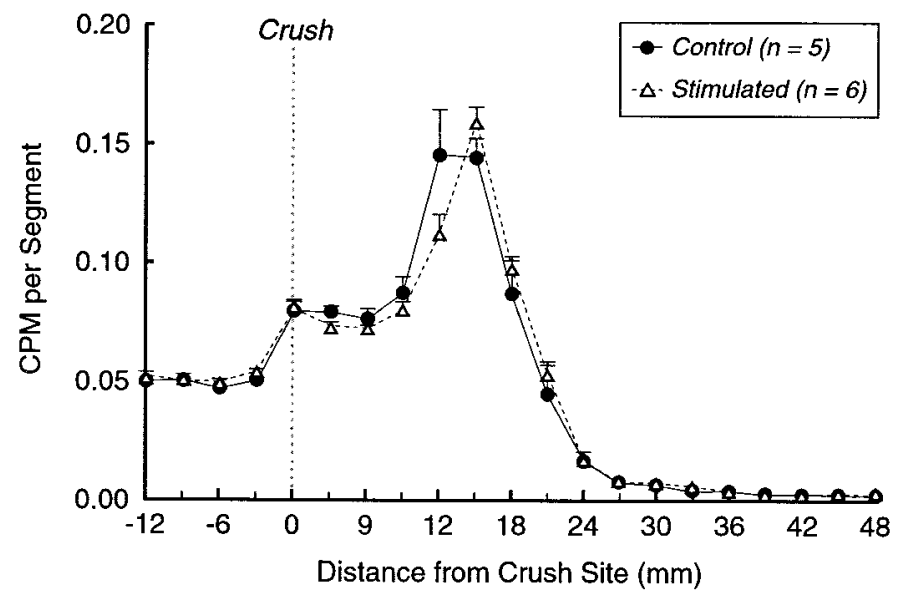

Figure 7. Conditioning trials-radiotracer. Sciatic nerve stimulation was followed in 1 week by crush. Label was injected $7 \mathrm{~d}$ after crush, and specimens were counted $1 \mathrm{~d}$ later. These animals do not differ significantly from the $8 \mathrm{~d}$ regeneration speed controls, and thus provide no evidence for a conditioning effect.

cross the suture line by up to 2 weeks after suture. Similar findings were obtained when regenerating nerves were studied longitudinally with a vibrating probe (Kerns et al., 1991). Evidence of staggered regeneration has thus resulted from those studies capable of detecting it.

Regeneration stagger is observed most readily by sequential application of retrograde tracing to identify neurons that project to a given area of nerve. Neurons that do not reach the site of labeling will not be counted, whereas those that project multiple sprouts will still be identified only once. Sequential evaluation of rat femoral nerve repair with distal labeling identifies only those motoneurons that have crossed the repair and penetrated the distal stump by $2.5 \mathrm{~cm}$. Using this technique, we found the number of motoneurons correctly reinnervating the quadriceps muscle branch to increase progressively from two to 10 weeks (Brushart, 1993; Al-Majed et al., 2000b). Our interest in regeneration stagger was sparked by the observation that it could be temporally compressed by application of $20 \mathrm{~Hz}$ electrical stimulation to the site of nerve repair for $1 \mathrm{hr}$ during surgery (Al-Majed et al., 2000b). The results of distal labeling 3 weeks after stimulation and repair equaled those requiring 8-10 weeks after suture without stimulation.

The mechanism by which electrical stimulation hastens regeneration to its completion is not revealed by distal labeling. We have thus separately analyzed the effects of stimulation on initial reinnervation of the distal stump and on the speed of axon elongation within the distal stump environment. The technique of proximal labeling (Fig. 1) was developed to identify axons as soon as possible after they have crossed the repair. Its accuracy was established by proximal labeling controls. The femoral nerve was injected with tracer immediately after suture, confirming that, under the experimental conditions, significant amounts of FR did not diffuse the $1.5 \mathrm{~mm}$ between injection and repair sites. The repair crossing experiments applied proximal labeling to the evaluation of femoral nerve regeneration $4 \mathrm{~d}$, 1 week, 2 weeks, 3 weeks, and 4 weeks after suture. Significantly more motoneurons crossed the repair in stimulated animals at all time periods until the results finally converged at 4 weeks (Fig. 2). A more meaningful picture of regeneration stagger is obtained by quantifying the new "crossing events" that occur during the intervals between labeling (Fig. 3). This approach revealed that electrical stimulation recruits many motoneurons to regenerate across the suture line and penetrate the distal stump earlier than they normally would. Whether quiescent motoneurons have been forced more rapidly into their regeneration program or whether this program has been modified to facilitate crossing the repair gap remains to be determined.

\section{Regeneration speed}

Electrical stimulation could also modify regeneration by increasing the speed of axon elongation within the distal stump. To evaluate this possibility, we performed radiolabeling studies of fast axoplasmic transport either 4 or $8 \mathrm{~d}$ after sciatic crush with or without electrical stimulation. Previous measurements of motor axon regeneration speed have relied on radiotracer evaluation at sequential times after crush, plotting distance versus time to determine speed (Forman and Berenberg, 1978; Danielsen et al., 1986). In contrast, we have studied 10 nerves at 4 d (5 crush, 5 crush plus stimulation) and 10 at $8 \mathrm{~d}$ (5 crush, 5 crush plus stimulation) (Fig. 4), characterizing each nerve by the distance from the crush at which the level of radioactivity in the leading edge of the curve fell to $50 \%$ of the mean peak value. This measure should be more precise than characterization of the front of regeneration by the drop-off to background at the leading edge of the curve (Forman and Berenberg, 1978), a point more easily influenced by the sampling interval. Subtraction of the $4 \mathrm{~d}$ mean from the $8 \mathrm{~d}$ mean will then provide a precise measure of the distance covered in $4 \mathrm{~d}$. Using this technique, we determined that motor axon regeneration occurred at a speed of $3.38 \pm 0.58$ $\mathrm{mm} / \mathrm{d}$ with stimulation and $3.27 \pm 0.90 \mathrm{~mm} / \mathrm{d}$ without stimulation. One hour of electrical stimulation at the time of nerve crush thus had no significant effect on the speed of axon propagation 
within the distal nerve stump. These rates are consistent with previous findings (Forman and Berenberg, 1978; Danielsen et al., 1986).

We also studied regeneration speed after femoral nerve transection and repair by using anterograde transport of neurobiotin to label advancing axons in the distal stump (Fig. 5). Stimulation did not significantly alter the distance traveled by regenerating axons $(p=0.4298)$. Our morphologic findings thus corroborate those based on the measurement of axoplasmic transport.

\section{Conditioning}

Although electrical stimulation immediately after crush had no effect on the speed of motor axon regeneration, it still might condition the neuron to respond to subsequent injury with an increased rate of elongation. Such an effect is produced by preinjury application of PEMF (Sisken et al., 1989; Kanje et al., 1993) as well as by relatively subtle injuries such as vibration (Dahlin et al., 1992), mild compression (Dahlin and Kanje, 1992), and inflammation near the cell body (Dahlin, 1992). Rat sciatic nerve was subjected to trial conditioning stimulation for $1 \mathrm{hr}$, followed in 1 week by either crush or transection and repair. This pre-injury stimulation changed neither the speed of axon regeneration, nor the number of motoneurons correctly reinnervating the muscle branch or incorrectly reinnervating the cutaneous branch. We are thus unable to provide evidence that stimulation conditions the neuron to respond to crush with increased regeneration speed or to transection and repair with more rapid development of regeneration specificity. These findings strongly suggest that stimulation in the absence of axonal injury does little to alter the future behavior of the motoneuron.

\section{Potential mechanisms}

Recent experiments with the rat femoral nerve model demonstrate that electrical stimulation affects the cell body directly and suggest that brain-derived neurotrophic factor (BDNF) is involved. Blocking action potential transmission from periphery to neuron by application of a tetrodotoxin cuff during stimulation abolished the stimulation effect, confirming involvement of the cell body (Al-Majed et al., 2000b). Participation of BDNF in this process is strongly supported by in situ hybridization studies of motoneurons that reveal early, dramatic upregulation of mRNA encoding BDNF and TrkB in response to stimulation (Al-Majed et al., 2000a). This observation is consistent with evidence linking electrical activity and BDNF expression in vitro (Gosh et al., 1994) and in vivo (Castren et al., 1992), and implicating BDNF in the stimulation of both CNS (Kobayashi et al., 1997; Menei et al., 1998) and PNS (Utley et al., 1996; Lewin et al., 1997; Kohmura et al., 1999; Zhang et al., 2000) regeneration.

Upregulation of BDNF by electrical stimulation is likely to involve both calcium and cAMP. $\mathrm{Ca}^{2+}$ influx couples electrical activity to subsequent gene expression in several systems (Finkbeiner and Greenberg, 1998). The initial calcium flux may be amplified by calcium-induced calcium release (Kocsis et al., 1994) to activate the cAMP response element-binding protein (CREB) (Grewal et al., 2000). In the specific case of BDNF, $\mathrm{Ca}^{2+}$ signaling is propagated to the CREB-mediated component of BDNF expression by CaM kinase IV (Shieh and Ghosh, 1999). Although both BDNF and TrkB are produced intraneurally, they are both rapidly externalized in response to membrane depolarization (Meyer-Franke et al., 1998; Balkowiec and Katz, 2000), opening the possibility of receptor-mediated autocrine signaling. The specific pathways through which BDNF plus TrkB might then pro- mote regeneration are less clear (Goldberg and Barres, 2000), potentially including the extracellular signal-regulated kinase signaling cascade (Perron and Bixby, 1999) or the Akt pathway (Namikawa et al., 2000). Another consequence of electrically mediated $\mathrm{Ca}^{2+}$ influx is an increase in intracellular cAMP (Hempel et al., 1996; Shen et al., 1999) through activativation of $\mathrm{Ca}^{2+}$-dependent adenylate cyclase (Xia and Storm, 1997). Elevated intracellular cAMP is a potent stimulator of axonal regeneration in vivo (Pichichero et al., 1973; Gershenbaum and Roisen, 1980; Kilmer and Carlsen, 1984; Carlsen et al., 1987). It may promote regeneration by directly activating downstream pathways through PKA (Cai et al., 1999) or by helping axons to overcome the inhibitory effects of MAG in the distal pathway (Schafer et al., 1996; Torigoe and Lundborg, 1998; Cai et al., 1999; Ming et al., 2001).

\section{Implications}

This study has implications for both the quantitative assessment of regeneration and the formulation of strategies to improve clinical outcome. Most previous efforts to quantify regeneration were not equipped to detect variability in the timing of distal stump reinnervation. However, serial examination with radiolabeled proteins (Danielsen et al., 1986) and axon tracing techniques (Al-Majed et al., 2000b) suggested a more complex picture. Identification of motoneurons as soon as they project across the repair confirms and expands these results; whereas some motor axons enter the distal stump in $4 \mathrm{~d}$, others require up to 4 weeks. Regeneration stagger is thus a morphologically significant attribute of nerve repair that should be considered in future studies. The substantial effects of electrical stimulation in compressing regeneration stagger suggest its use as a clinical tool. However, stimulation has no effect on the speed of axon elongation. A combined approach will most likely be required, with a "start-up" technique such as electrical stimulation, followed by treatments directed at the neuron and growth cone to speed regeneration, at the pathway to maintain responsiveness of denervated Schwann cells and at the end organs to maintain their viability.

\section{REFERENCES}

Abercrombie M (1946) Estimation of nuclear population from microtome sections. Anat Rec 94:239-247.

Al-Majed AA, Brushart TM, Gordon T (2000a) Electrical stimulation accelerates and increases expression of BDNF and trkB rnRNA in regenerating rat femoral motoneurons. Eur J Neurosci 12:4381-4390.

Al-Majed AA, Neumann CM, Brushart TM, Gordon T (2000b) Brief electrical stimulation promotes the speed and accuracy of motor axonal regeneration. J Neurosci 20:2602-2608.

Balkowiec A, Katz DM (2000) Activity-dependent release of endogenous brain-derived neurotrophic factor from primary sensory neurons detected by ELISA in situ. J Neurosci 20:7417-7423.

Brushart TM (1988) Preferential reinnervation of motor nerves by regenerating motor axons. J Neurosci 8:1026-1031.

Brushart TM (1990) Preferential motor reinnervation: a sequential double-labeling study. Restor Neurol Neurosci 1:281-287.

Brushart TM (1993) Motor axons preferentially reinnervate motor pathways. J Neurosci 13:2730-2738.

Brushart TM, Gerber J, Kessens P, Chen YG, Royall RM (1998) Contributions of pathway and neuron to preferential motor reinnervation. J Neurosci 18:8674-8681.

Cai DM, Shen YJ, De Bellard M, Tang S, Filbin MT (1999) Prior exposure to neurotrophins blocks inhibition of axonal regeneration by MAG and myelin via a cAMP-dependent mechanism. Neuron 22:89-101.

Carlsen R, De Leon M, Tetzlaff W, Swedberg E, Parhad I, Bisby M (1987) Expression of alpha-tubulin mRNA is increased by cAMP in vitro. Fed Proc 46:522.

Castren E, Zafra F, Thoenen H, Lindholm D (1992) Light regulates expression of brain-derived neurotrophic factor mRNA in rat visual cortex. Proc Natl Acad Sci USA 89:9444-9448. 
Dahlin L (1992) Stimulation of regeneration of the sciatic nerve by experimentally induced inflammation in rats. Scand J Plast Reconstr Hand Surg 26:121-125.

Dahlin L, Kanje M (1992) Conditioning effect induced by chronic nerve compression. Scand J Plast Reconstr Hand Surg 26:37-41.

Dahlin L, Necking L, Lundstrom R, Lundborg G (1992) Vibration exposure and conditioning lesion effect in nerves: An experimental study in rats. J Hand Surg 17A:858-861.

Danielsen N, Lundborg G, Frizell M (1986) Nerve repair and axonal transport: outgrowth delay and regeneration rate after transection and repair of rabbit hypoglossal nerve. Brain Res 376:125-132.

Du Bois-Reymond E (1848) Untersuchungen uber Thierische Elektricitat. Berlin: Verlag.

Duchenne de Boulogne (1855) De L'Electrisation Localise, et de son Application a La Physiologie, a La Pathologie et a La Therapeutique. Paris: Bailliere.

Finkbeiner S, Greenberg M (1998) $\mathrm{Ca}^{2+}$ channel-regulated neuronal gene expression. J Neurobiol 37:171-189.

Forman D, Berenberg R (1978) Regeneration of motor axons in the rat sciatic nerve studied by labeling with axonally transported radioactive proteins. Brain Res 156:213-225.

Forman DS, Wood DK, DeSilva S (1979) Rate of regeneration of sensory axons in transected rat sciatic nerve repaired with epineurial sutures. J Neurol Sci 44:55-59.

Gershenbaum M, Roisen F (1980) The effects of dibutyryl cyclic adenosine monophosphate on the degeneration and regeneration of crushlesioned rat sciatic nerve. Neuroscience 5:1565-1580.

Goldberg JL, Barres BA (2000) The relationship between neuronal survival and regeneration. Annu Rev Neurosci 23:579-612.

Gosh A, Carnahan J, Greenberg M (1994) Requirement for BDNF in activity-dependent survival of cortical neurons. Science 263:1618-1623.

Grewal S, Fass D, Yao H, Ellig C, Goodman R, Stork P (2000) Calcium and cAMP signals differentially regulate cAMP-responsive elementbinding function via a Rap1-extracellular signal-regulated kinase pathway. J Biol Chem 275:34433-34441.

Hempel C, Vincent P, Adams S, Tsien R, Selverston A (1996) Spatiotemporal dynamics of cyclic AMP signals in an intact neural circuit. Nature 384:166-169.

Hinkle L, McCaig CD, Robinson KR (1981) The direction of growth of differentiating neurons and myoblasts from frog embryos in an applied electric field. J Physiol (Lond) 314:121-135.

Holmquist B, Kanje M, Kerns J, Danielsen N (1993) A mathematical model for regeneration rate and initial delay following surgical repair of peripheral nerves. J Neurosci Methods 48:27-33.

Jacquin MF, Hu JW, Sessle BJ, Renehan WE, Waite PM (1992) Intraaxonal neurobiotin injection rapidly stains the long-range projections of identified trigeminal primary afferents in vivo: comparisons with HRP and PHA-L. J Neurosci Methods 45:71-86.

Jenq C-B, Coggeshall RE (1984) Regeneration of axons in tributary nerves. Brain Res 310:107-121.

Kanje M, Rusovan A, Sisken B, Lundborg G (1993) Pretreatment of rats with pulsed electromagnetic fields enhances regeneration of the sciatic nerve. Bioelectromagnetics 14:353-359.

Kerns JM, Lucchinetti C (1992) Electrical field effects on crushed nerve regeneration. Exp Neurol 117:71-80.

Kerns JM, Fakhouri AJ, Weinrib HP, Freeman JA (1991) Electrical stimulation of nerve regeneration in the rat: the early effects evaluated by a vibrating probe and electron microscopy. Neuroscience 40:93-107.

Kerns JM, Pavkovic IM, Fakhouri AJ, Gray GT (1994) Electrical stimulation of nerve regeneration in the rat: Functional evaluation by a twitch tension method. Restor Neurol Neurosci 6:175-180.

Kilmer S, Carlsen R (1984) Forskolin activation of adenylate cyclase in vivo stimulates nerve regeneration. Nature 307:455-457.

Kobayashi NR, Fan DP, Giehl KM, Bedard AM, Wiegand SJ, Tetzlaff W (1997) BDNF and NT-4/5 prevent atrophy of rat rubrospinal neurons after cervical axotomy, stimulate GAP-43 and T 1-tubulin mRNA expression, and promote axonal regeneration. J Neurosci 17:9583-9595.

Kocsis JD, Rand MN, Lankford KL, Waxman SG (1994) Intracellular calcium mobilization and neurite outgrowth in mammalian neurons. J Neurobiol 25:252-264.

Kohmura E, Yuguchi T, Yoshimine T, Fujinaka T, Koseki N, Sano A, Kishino A, Nakayama C, Sakaki T, Nonaka M, Takemoto O, Hayakawa $\mathrm{T}$ (1999) BDNF atelocollagen mini-pellet accelerates facial nerve regeneration. Brain Res 849:235-238.
Lasek RJ (1968) Axoplasmic transport of labeled proteins in rat ventral motor neurons. Exp Neurol 21:41-51.

Lewin SL, Utley DS, Cheng ET, Verity AN, Terris DJ (1997) Simultaneous treatment with BDNF and CNTF after peripheral nerve transection and repair enhances rate of functional recovery compared with BDNF treatment alone. Laryngoscope 107:992-999.

Mackinnon S, Dellon L, O'Brien J (1991) Changes in nerve fiber numbers distal to a nerve repair in the rat sciatic nerve model. Muscle Nerve 14:1116-1122.

Menei P, Montero-Menei C, Whittemore SR, Bunge RP, Bunge MB (1998) Schwann cells genetically modified to secrete human BDNF promote enhanced axonal regrowth across transected adult rat spinal cord. Eur J Neurosci 10:607-621.

Meyer-Franke A, Wilkinson G, Kruttgen A, Hu M, Munro E, Hanson M, Reichardt L, Barres B (1998) Depolarization and cAMP elevation rapidly recruit TrkB to the plasma membrane of CNS neurons. Neuron 21:681-693.

Ming GL, Henley J, Tessier-Lavigne M, Song HJ, Poo MM (2001) Electrical activity modulates growth cone guidance by diffusible factors. Neuron 29:441-452.

Namikawa K, Honma M, Abe K, Takeda M, Mansur K, Obata T, Miwa A, Okado H, Kiyama H (2000) Akt/Protein kinase B prevents injuryinduced motoneuron death and accelerates axonal regeneration. J Neurosci 20:2875-2886.

Nix W, Hopf H (1983) Electrical stimulation of regenerating nerve and its effect on motor recovery. Brain Res 272:21-25.

Orgel MG, O'Brien WJ, Murray HM (1984) Pulsing electromagnetic field therapy in nerve regeneration: an experimental study in the cat. Plast Reconstr Surg 73:173-182.

Patel N, Poo M-M (1982) Orientation of neurite growth by extracellular electric fields. J Neurosci 2:483-496.

Perron J, Bixby J (1999) Distinct neurite outgrowth signaling pathways converge on ERK activation. Mol Cell Neurosci 13:362-378.

Pichichero M, Beer B, Clody D (1973) Effects of dibutyryl cyclic AMP on restoration of function of damaged sciatic nerve in rats. Science 182:724-725.

Pockett S, Gavin RM (1985) Acceleration of peripheral nerve regeneration after crush injury in the rat. Neurosci Lett 59:221-224.

Politis MJ, Zanakis MF, Albala BJ (1988) Facilitated regeneration in the rat peripheral nervous system using applied electric fields. J Trauma 28:1375-1381.

Rusovan A, Kanje M, Mild KH (1992) The stimulatory effect of magnetic fields on regeneration of the rat sciatic nerve is frequency dependent. Exp Neurol 117:81-84.

Schafer M, Fruttiger M, Montag D, Schachner M, Martini R (1996) Disruption of the gene for the myelin-associated glycoprotein improves axonal regrowth along myelin in $\mathrm{C} 57 \mathrm{BL} / \mathrm{Wld}$ mice. Neuron 16:1107-1113.

Scherer SS, Easter SS (1984) Degenerative and regenerative changes in the trochlear nerve of goldfish. J Neurocytol 13:519-565.

Shen S, Wiemelt A, McMorris FA, Barres B (1999) Retinal ganglion cells lose trophic responsiveness after axotomy. Neuron 23:285-295.

Shieh PB, Ghosh A (1999) Molecular mechanisms underlying activitydependent regulation of BDNF expression. J Neurobiol 41:127-134.

Sisken BF, Kanje M, Lundborg G, Herbst E, Kurtz W (1989) Stimulation of rat sciatic nerve regeneration with pulsed electromagnetic fields. Brain Res 485:309-316.

Torigoe K, Lundborg G (1998) Selective inhibition of early axonal regeneration by myelin-associated glycoprotein. Exp Neurol 150:254-262.

Utley DS, Lewin SL, Cheng ET, Verity AN, Sierra D, Terris DJ (1996) Brain-derived neurotrophic factor and collagen tubulization enhance functional recovery after peripheral nerve transection and repair. Arch Otolaryngol Head Neck Surg 122:407-413.

Xia Z, Storm D (1997) Calmodulin-regulated adenylyl cyclases and neuromodulation. Curr Opin Neurobiol 7:391-396.

Zhang JY, Luo XG, Xian CJ, Liu ZH, Zhou XF (2000) Endogenous $\mathrm{BDNF}$ is required for myelination and regeneration of injured sciatic nerve in rodents. Eur J Neurosci 12:4171-4180.

Zienowicz RJ, Thomas BA, Kurtz WH, Orgel MG (1991) A multivariate approach to the treatment of peripheral nerve transection injury: the role of electromagnetic field therapy. Plast Reconstr Surg 87:122129. 